\title{
AHP in nonlinear scaling: from two-envelope problem to modeling by predictors
}

\author{
Stan Lipovetsky ${ }^{\text {a* }}$ \\ alndependent researcher, Minnetonka, MN, United States \\ *stan.lipovetsky@gmail.com
}

\begin{abstract}
Paper aims: Extending the AHP abilities to new areas from explaining the famous Two-Envelope paradox to finding priorities as functions of auxiliary variables is suggested.

Originality: The proposed innovative and advantageous approach permits to find the non-constant priorities via regression modeling for the exponential or Cobb-Douglas production functions.
\end{abstract}

Research method: Transforming the ratio scale into the additive or logarithmic scales, which correspond to the multiplicative utility function, opens new possibilities in the multiple-criteria decision making.

Main findings: Two-Envelop problem is resolved, priority vectors are presented as functions by auxiliary variables, preferences are predicted and profiled by predictors.

Implications for theory and practice: The suggested techniques open novel possibilities of multiple-criteria decisions helping to researchers and managers to discover useful features of priority behavior due to the demographic, socioeconomic, or technical properties of the compared alternatives, and to find optimal solutions for individual customers.

Keywords

AHP. Two-envelope paradox. Nonlinear scaling. Multiplicative utility. Priority as function. Exponential function. CobbDouglas production function.

How to cite this article: lipovetsky, S. (2021). AHP in nonlinear scaling: from two-envelope problem to modeling by predictors. Production, 31, e20210007. https://doi.org/10.1590/0103-6513.20210007.

Received: Feb. 09, 2021; Accepted: Mar. 31, 2021.

\section{Introduction}

The work extends some ideas from the Analytic Hierarchy Process (AHP) to the new areas, from resolving the so-called Two-Envelopes paradox (let us denote it 2EP) to building the priorities as functions by auxiliary predictors. The fascinating 2EP problem has been studied in multiple works based on probability theory (Abbott et al., 2010), decision making (Egozcue \& Fuentes García, 2015), Bayesian evaluations (Albers et al., 2005), game theory (McDonnell et al., 2011), logic (Lee, 2013), epistemology (Markosian, 2011), and philosophy (Yi, 2013). Resolving this paradox via transformation of the ratio scale into the additive or logarithmic scales corresponding to using the multiplicative utility function is proposed in the work (Lipovetsky, 2020a) presented at the International Symposium on AHP of 2020. Besides the 2EP problem, the current paper describes new methods of building AHP priority vectors as functions by auxiliary predictors of influence.

The AHP is one of the main methods for solving various multiple-criteria decision making problems. It had been founded by Thomas Saaty (1980) and developed in numerous works (Saaty \& Vargas, 1984; Lootsma, 1999; Whitaker, 2007; Ishizaka et al., 2020; Neira-Rodado et al., 2020; Ramos et al., 2020; Yang et al., 2020; Lipovetsky, 2021). The AHP preferences can be calculated by different techniques using the pairwise comparison matrices to find the vectors of priorities, with the elements of these vectors commonly presented as constant values. In multi-person decision making (Saaty \& Peniwati, 2007; Lipovetsky, 2009), the experts' opinions can dependent 
on their different attitudes and tastes, demographic and socio-economic properties, many other characteristics influencing their choices.

The current paper explores new possibilities for building the priority vectors where each element is presented as a function obtained via regression modeling. The varying priorities can be predicted for individual respondents, profiled by each predictor, studied by the predictor importance, and estimated by the characteristic of significance and quality known in the regression modeling. For priority modeling by predictors, the dependency by exponent and Cobb-Douglas production functions have been tried. Numerical results show that the proposed methods reveal new and useful features of priority behavior. A possibility of finding personalized key drivers in regression analysis for individual respondents was studied in (Lipovetsky, 2020b), and the current work demonstrates that AHP priorities can also be estimated for each of the respondents in the group of experts. The novelty in considering AHP priorities not as constants but as dependencies on the auxiliary predictors of socio-economic environment, demography and any other characteristics of influence can noticeably extend the AHP abilities and applications for solving numerous multiple-criteria decision making problems.

The paper is organized as follows: Section 11 describes AHP in resolving the Two-Envelope paradox, Section 111 extends the priorities from constants to functions by the predictors. Section IV discusses numerical example, and Section V summarizes.

\section{Two-envelope problem in nonlinear scaling and multiplicative utility}

Two-Envelope paradox (2EP) in its main version is as follows. The player is presented with two envelopes and told that one of them contains twice as much money as the other envelope. The player takes one of them without looking inside, and then has the option to take another envelope instead of the first one. It seems that without any information of the content the player can value both envelopes equally, so there is no reason to substitute one with another. However, assuming that the $1^{\text {st }}$ envelope contains a value $A$, then the $2^{\text {nd }}$ can have $2 A$ or $A / 2$ equally probable, and its expected value equals the mean

$$
(2 A+0.5 A) / 2=1.25 A
$$

which is $25 \%$ profit from switching envelopes. It is a great result, but we could denote the amount in the $2^{\text {nd }}$ envelope as $A$ and repeat the derivation getting $1.25 A$ already in $1^{\text {st }}$ envelope, as if each of them is worth more than the other one. So, to switch or not to switch? - That is the question.

Measuring values in the second envelope as two times larger or smaller than in the first envelope, actually corresponds to the ratio scale used in the AHP. However, a ratio scale is not additive, and if the summing is performed in the ratio scale it could easily produce unclear or strange results, exactly as it happens in the 2EP. Resolution of this problem can be achieved by transforming the ratio scale into another scale which permits the operation of addition. This transformation can be done with the share and logarithm of the quotients. In such nonlinear scales, the expected value equals zero, and the corresponding expected value in the original scale equals one. It shows that there is no gain in changing envelopes, and the paradox is dissolved. Let us describe these scales in more detail.

The ratio scales are well-known in some multiple-criteria decision making (MCDM) methods. Particularly, the AHP is based on the data elicited via comparisons of alternatives made in the ratio scale of how many times one item is preferred over another one. If one item is $K$ times preferred over another one, then the ratio of their preferences equals $K$. The value $K$ is measured by the natural numbers from 1 when the items are equal, 2 for a slight preference, etc., up to 9 of the overwhelming preference. If a preferences' ratio of the $j$-th to $k$-th item equals $K$ then the opposite ratio of the $k$-th to $j$-th item equals the reciprocal value $1 / K$. After eliciting the pairwise ratios from an expert, the matrix of the pairwise comparisons can be built with the elements $a_{j k}$ corresponding to the ratio of priorities of the $j$-th to $k$-th items. The matrix elements are reciprocally transposed, so $a_{j k}=1 / a_{k j}$, for instance, if $a_{j k}=2$ then $a_{k j}=0.5$, or if $a_{j k}=8$ then $a_{k j}=0.125$. The bigger values have a bigger impact in numerical estimations performed in the regular additive scale. The classical AHP solves the eigenproblem of such a matrix, and its first eigenvector serves as the vector of the items' priorities. The results of priority estimation could be very sensitive to change in the data because of the unbalanced impact of the bigger ratios and their small reciprocal counterparts.

In the study (Lipovetsky \& Conklin, 2015a), it was suggested to transform the matrix of pairwise ratios into the matrix of their shares, and it was shown that such a robust matrix can be obtained via the Markov chain modeling. Employing the idea of reciprocal numbers' conversion into the balanced additive scale for the 
problem of two envelopes, let us consider the transformation of a value $K$ and $1 / K$ into their shares $S_{K}$ and $S_{1 / K}$, respectively. It can be done by the following formulae:

$$
S_{K}=\frac{1}{1+\frac{1}{K}}=\frac{K}{K+1} ; \quad S_{1 / K}=\frac{1}{1+\frac{1}{1 / K}}=\frac{1}{K+1} ; \quad S_{K}+S_{1 / K}=1
$$

For $K=2$ and $1 / K=0.5$, the shares of these values by (2) are:

$$
S_{K=2}=\frac{1}{1+\frac{1}{2}}=\frac{2}{3} ; \quad S_{\frac{1}{K}=0.5}=\frac{1}{1+\frac{1}{1 / 0.5}}=\frac{1}{3} ; \quad S_{2}+S_{0.5}=1
$$

For $K=1$, as in the first original envelope's value, the transformation (2) yields the share

$$
S_{K=1}=\frac{1}{1+\frac{1}{1}}=\frac{1}{2}
$$

The deviations $D$ of the share values (2) from the basic level (4) are as follows:

$$
D S_{K}=\frac{K}{K+1}-\frac{1}{2}=\frac{K-1}{2(K+1)} ; \quad D S_{\frac{1}{K}}=\frac{1}{K+1}-\frac{1}{2}=\frac{1-K}{2(K+1)} ; \quad D S_{K}+D S_{1 / K}=0
$$

with total of the centered shares equals zero.

For $K=2$, the deviations (5) of the shares (3) from the basic level (4) are:

$$
D S_{K=2}=\frac{2-1}{2(2+1)}=\frac{1}{6} ; \quad D S_{\frac{1}{K}=0.5}=\frac{1-2}{2(2+1)}=-\frac{1}{6} ; \quad D S_{K=2}+D S_{\frac{1}{K}=0.5}=0
$$

The expected utility with equal probabilities, or the mean value of the shares calculated by the nonlinear scale, due to the last relation in (3) is:

$$
0.5 S_{2}+0.5 S_{0.5}=\frac{1}{2}
$$

which equals the basic level (4). It coincides with the utility in the first envelope, and no substitution of the envelopes changes this utility. Therefore, in the adequate scale of shares for measuring utility, the 2EP problem dissolves.

In the multiplicative AHP (Saaty \& Vargas, 1984) the pairwise ratios are transformed by logarithm, so the reciprocal values $K$ and $1 / K$ become the opposite numbers:

$$
\log (K)=-\log (1 / K)
$$

These logarithms are equal by the absolute value, so they are comparable in the additive scale. Applications of additive and multiplicative modes and their comparison have been studied in various problems, and transforming to logarithmic scales corresponds to using the multiplicative utility function known in decision theory (for example, Keeney \& Raiffa, 1993; Noto et al., 2020).

For $K=2$ and $1 / K=0.5$, the expected utility with equal probabilities, or the mean value in the logarithmic scale (8), equals:

$$
0.5 \log (2)+0.5 \log \left(\frac{1}{2}\right)=0
$$

As $\log (1)=0$, the expected utility in the original scale equals 1 , which is the value in the first envelope with the amount $A(1)$. This conclusion can be reached if to estimate the expected utility not by the arithmetic mean 
but by the geometric mean which is an adequate measure for the multiplicative utility. For 2EP with equal probability of choice between the envelopes with $2 \mathrm{~A}$ and $0.5 \mathrm{~A}$ values, the expected value for the multiplicative utility equals:

$$
(2 A)^{0.5}(0.5 A)^{0.5}=\sqrt{(2 A)(0.5 A)}=A
$$

which coincides with the value in the original envelope. Thus, no substitution of the envelope changes the original utility, and in the adequate logarithmic scale for measuring utility in the multiplicative mode the paradox is resolved.

Comparing the shares and logarithmic scales, we can note that they behave very similarly and differ by a constant term depending on the base of logarithm. Table 1 presents examples of share and logarithmic transformations. The ratio $K$ in the first column of Table 1 is taken from 1 to 9 and the corresponding reciprocal values. The next two columns show the values of the centered shares (5) and logarithms (8) taken by the base 100. The last three columns present the quotient log/shares with values around 1 , the deviations values of $\log$-shares, and the absolute deviation values of $\mid \log$-shares $\mid$. In the last two bottom rows there are the mean values and standard deviations (std) of the values in each column, and all of those are very small. The results are mostly the same for a larger span of the ratios as well.

Shares and logarithm values from Table 1 profiled by the $K$ ratio values are presented in Figure 1, that shows that both curves behave very similarly. Indeed, the coefficient of pair correlation between the shares and logarithms equals 0.997 , which is a very high level of connection, and it does not depend on the base of logarithm.

Table 1. Share and logarithmic transformations of the ratio.

\begin{tabular}{|c|c|c|c|c|c|}
\hline ratio $\mathrm{K}$ & Shares (5) & Logarithm (8) & $\log /$ shares & log-shares & abs(log-shares) \\
\hline $1 / 9$ & -0.400 & -0.477 & 1.193 & -0.077 & 0.077 \\
\hline $1 / 8$ & -0.389 & -0.452 & 1.161 & -0.063 & 0.063 \\
\hline $1 / 7$ & -0.375 & -0.423 & 1.127 & -0.048 & 0.048 \\
\hline $1 / 6$ & -0.357 & -0.389 & 1.089 & -0.032 & 0.032 \\
\hline $1 / 5$ & -0.333 & -0.349 & 1.048 & -0.016 & 0.016 \\
\hline $1 / 4$ & -0.300 & -0.301 & 1.003 & -0.001 & 0.001 \\
\hline $1 / 3$ & -0.250 & -0.239 & 0.954 & 0.011 & 0.011 \\
\hline $1 / 2$ & -0.167 & -0.151 & 0.903 & 0.016 & 0.016 \\
\hline 1 & 0.000 & 0.000 & & 0.000 & 0.000 \\
\hline 2 & 0.167 & 0.151 & 0.903 & -0.016 & 0.016 \\
\hline 3 & 0.250 & 0.239 & 0.954 & -0.011 & 0.011 \\
\hline 4 & 0.300 & 0.301 & 1.003 & 0.001 & 0.001 \\
\hline 5 & 0.333 & 0.349 & 1.048 & 0.016 & 0.016 \\
\hline 6 & 0.357 & 0.389 & 1.089 & 0.032 & 0.032 \\
\hline 7 & 0.375 & 0.423 & 1.127 & 0.048 & 0.048 \\
\hline 8 & 0.389 & 0.452 & 1.161 & 0.063 & 0.063 \\
\hline 9 & 0.400 & 0.477 & 1.193 & 0.077 & 0.077 \\
\hline mean & 0.000 & 0.000 & 1.060 & 0.000 & 0.031 \\
\hline std & 0.330 & 0.363 & 0.098 & 0.042 & 0.026 \\
\hline
\end{tabular}

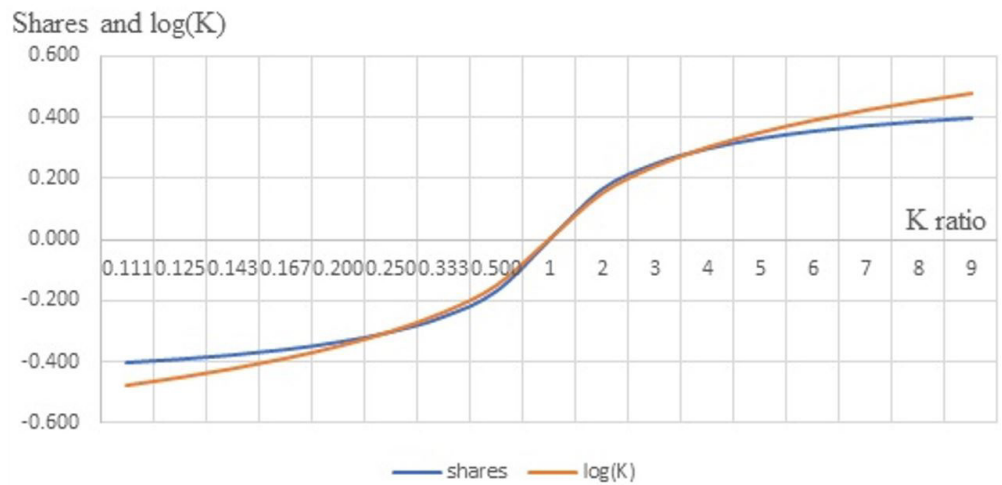

Figure 1. Shares and logarithm transformation profiled by the ratio $\mathrm{K}$. 


\section{AHP priority vectors as functions of auxiliary predictors}

In the classical AHP, a pairwise judgement $a_{i k}$ elicited from an expert presents a quotient of preference of the $j$-th item over $k$-th item in their comparison among all $n$ items. It corresponds to the theoretical ratio of the unknown priorities $w_{j}$ and $w_{k}$. In the case of exact estimates, the relation between the observed and theoretical ratios can be written in the equalized form:

$$
a_{j k}=w_{j} / w_{k}
$$

For the real data, the relations (11) should be represented in a model with deviations from the exact equalities. Assuming a multiplicative error term, the relation (11) can be represented as follows:

$$
a_{j k}=\frac{\alpha_{j}}{\alpha_{k}}\left(1+\delta_{j k}\right)
$$

where alpha $\alpha_{j}$ denotes the estimates for the elements of the theoretical priority vector $w$, and delta $\delta_{j k}$ are the relative errors. The relations (12) can be linearized by logarithmic transformation, and the least squares (LS) minimization of the relative deviations can be applied for estimation of the priorities:

$$
L S=\sum_{j, k=1}^{n}\left(\ln \left(1+\delta_{j k}\right)\right)^{2}=\sum_{j, k=1}^{n}\left(\ln a_{j k}-\ln \alpha_{j}+\ln \alpha_{k}\right)^{2}
$$

For small values $\delta_{j k} \ll 1$, the approximation exists $\ln \left(1+\delta_{j k}\right)=\delta_{j k}$, that means that criterion (13) corresponds to the relative errors squared. Minimizing by the logarithm of priorities $\ln \alpha_{j}$ produces the logarithmic least squares method also known as multiplicative solution with priorities presented by the geometric means in the rows of the AHP matrix. It corresponds to the nonlinear scaling in logarithmic transformation used in expressions (8)-(10). More detail on this solution is given in (Saaty \& Vargas, 1984; Lootsma, 1999; Lipovetsky \& Tishler, 1999; Lipovetsky, 2020c). The obtained priority vector is usually standardized in AHP by the total of its elements equals one:

$$
p_{j}=\frac{\alpha_{j}}{\sum_{k=1}^{n} \alpha_{k}}
$$

The group decision making includes multiple experts whose opinions can vary by their demographic, socioeconomics, technical and other functional characteristics impacting the pairwise evaluations. The more complicated data and problems require more complex models, and the known methods should be extended to incorporating auxiliary variables explaining variability of the priorities across the respondents with their specific features.

Several possible ways of extension of the constant AHP priorities to the more complex methods with priorities' predictors were considered in (Lipovetsky, 2021). Let us describe them briefly, then continue this investigation with new techniques.

Convenient functional forms for positive theoretical priorities $w_{j}$ in (11) are the exponents. Writing them in the alpha notations for the estimates, yields:

$$
\alpha_{j}^{i}=\exp \left(\alpha_{j 0}+\alpha_{j 1} x_{1}^{i}+\alpha_{j 2} x_{2}^{i}+\ldots+\alpha_{j M} x_{M}^{i}\right)
$$

where $x_{1}^{i}, x_{2}^{i}, \ldots, x_{M}^{i} X_{1 \text { i }}$, are the auxiliary predictors (with the subscript $m=1,2, \ldots, M$ - number of the variables; and the superscript $i=1,2, \ldots, N$ - number of the respondents); the $\alpha_{j 0}, \alpha_{j l}, \alpha_{j 2}, \ldots, \alpha_{j M}$ are the parameters of the $j$-th priority expressed via the intercept and the coefficients for linear combination of predictors. The model (12) with the constant parameters of preference $\alpha_{j}$ and $\alpha_{k}$ we extend now by the exponents (15):

$$
a_{j k}^{i}=\frac{\alpha_{j}^{i}}{\alpha_{k}^{i}}\left(1+\delta_{j k}^{i}\right)=\frac{\exp \left(\alpha_{j 0}+\alpha_{j 1} x_{1}^{i}+\alpha_{j 2} x_{2}^{i}+\ldots+\alpha_{j M} x_{M}^{i}\right)}{\exp \left(\alpha_{k 0}+\alpha_{k 1} x_{1}^{i}+\alpha_{k 2} x_{2}^{i}+\ldots+\alpha_{k M} x_{M}^{i}\right)}\left(1+\delta_{j k}^{i}\right)
$$

The pairwise ratios $a_{j k}^{i}$ and deviations $\delta_{j k}^{i}$ correspond to the comparison of $j$-th and $k$-th alternatives by the $i$-th respondent, and all other notations are the same as in the expression (15). In absence of the auxiliary 
$x$-variables, the model (16) reduces to just the intercept $a_{j 0}$, and with notations $a_{j}=\exp \left(a_{j 0}\right)$ it coincides with the constant priority model (12).

Applying to (16) the logarithmic transformation and LS criterion, similarly to (13) yields:

$$
\begin{gathered}
L S=\sum_{i=1 j}^{N} \sum_{j, k=1}^{n}\left(\ln \left(1+\delta_{j k}^{i}\right)\right)^{2} \\
=\sum_{i=1}^{N} \sum_{j, k=1}^{n}\left(\ln a_{j k}^{i}-\left(\alpha_{j 0}-\alpha_{k 0}\right)-\left(\alpha_{j 1}-\alpha_{k 1}\right) x_{1}^{i}-\ldots-\left(\alpha_{j M}-\alpha_{k M}\right) x_{M}^{i}\right)^{2}
\end{gathered}
$$

With the estimated parameters, the preference shares $p_{j k}^{i}$ in total (14) for each $i$-th respondent can be found by the expression coinciding with the multinomial-logit model:

$$
p_{j}^{i}=\frac{\exp \left(\alpha_{j 0}+\alpha_{j 1} x_{1}^{i}+\alpha_{j 2} x_{2}^{i}+\ldots+\alpha_{j M} x_{M}^{i}\right)}{\sum_{k=1}^{n} \exp \left(\alpha_{k 0}+\alpha_{k 1} x_{1}^{i}+\alpha_{k 2} x_{2}^{i}+\ldots+\alpha_{k M} x_{M}^{i}\right)}
$$

For uniqueness of solution in finding the odds ratio (16)-(17) or the multinomial-logit model (19) parameters, one set of the coefficients is commonly taken as the basic and put to zero, for instance, the first share's parameters equal zero. Such kind of models are widely used in the choice modeling (for example, lipovetsky, 2019). In practical applications, it is better to work with the exponential model (15)-(17) which employs fitting by all $n(n-1) / 2$ pairwise ratios and produces more precise estimates, in comparison with the multinomial-logit model (18) which fulfils fitting by only $n-1$ values related to the harmonic means of the paired ratios in the columns of the AHP matrix (more detail is given in Lipovetsky, 2021).

To continue research started in (Lipovetsky, 2021), let us consider other approaches to priority modeling by predictors. Instead of the priority ratio (11) we can use the priority paired shares used in (2)-(4). Theoretical priority shares $S_{j k}$ can be defined as $j$-th priority in the sum of $j$-th and $k$-th priorities, which corresponds to the following representation via the elicited ratios (11):

$$
S_{j k}=\frac{w_{j}}{w_{j}+w_{k}}=\frac{w_{j} / w_{k}}{1+w_{j} / w_{k}}=\frac{a_{j k}}{1+a_{j k}}=\frac{1}{1+a_{k j}}
$$

With the priorities defined as exponent functions of the predictor aggregates (15)-(17), the paired shares (19) can be presented as follows:

$$
S_{j k}=\frac{1}{1+\exp \left(\left(\alpha_{k 0}-\alpha_{j 0}\right)+\left(\alpha_{k 1}-\alpha_{j 1}\right) x_{l}^{i}+\ldots+\left(\alpha_{k M}-\alpha_{j M}\right) x_{M}^{i}\right)}
$$

This expression shows that the shares (19) correspond to the logistic functions. It can be used to fit the empirical data at the right-hand side (19), but the results of this modeling coincide with the obtained by the model (16), because logarithm of the odds of (20) produces the relation $\ln \left(S_{j k} /\left(1-S_{j k}\right)\right)=a_{j k}$ which is the same original paired ratio function (15).

Besides the exponential (16), multinomial (18), or logit (20) models, other functional forms can be tried. For the positive predictors, a multivariate Cobb-Douglas (CD) function for priority modeling can be applied. It is presented as follows:

$$
\alpha_{j}^{i}=e^{\alpha_{j 0}}\left(x_{1}^{i}\right)^{\alpha_{j 1}}\left(x_{2}^{i}\right)^{\alpha_{j 2}} \ldots\left(x_{M}^{i}\right)^{\alpha_{j M}}
$$

Logarithm of it transforms the CD function into the linearized form by the parameters:

$$
\ln \alpha_{j}^{i}=\alpha_{j 0}+\alpha_{j 1} \ln x_{1}^{i}+\alpha_{j 2} \ln x_{2}^{i}+\ldots+\alpha_{j M} \ln x_{M}^{i}
$$

It can be used instead of the exponential function (15) in the preference modeling by the auxiliary predictors. If to use logarithms of predictors in the exponential function (15) it reduces to CD function (21), then all relations (16)-(18) hold for it as well. 
The choice between the exponential (15) and CD (22) forms can be made in accordance with the interpretation of their parameters. A parameter $\alpha_{j m}$ of the predictor $x_{m}$ influence on the priority $\alpha_{j}$ in the total priority (15) is defined by the derivative of its logarithm:

$$
\frac{\partial \ln \alpha_{j}}{\partial x_{m}}=\frac{\partial \alpha_{j} / \alpha_{j}}{\partial x_{m}}=\alpha_{j m}
$$

It means the parameter $\alpha_{j m}$ equals the percent change of this priority $\partial \alpha_{j} / \alpha_{j}$ due to the unit change $\partial x_{m}$ in this predictor. In other words, each parameter $\alpha_{j m}$ in (15) defines the half-elasticity of the priority $\alpha_{j}$ due to the varied predictor $x_{m}$. In contrast, the parameter $\alpha_{j m}$ due to (22) is defined by the following derivative:

$$
\frac{\partial \ln \alpha_{j}}{\partial \ln x_{m}}=\frac{\partial \alpha_{j} / \alpha_{j}}{\partial x_{m} / x_{m}}=\alpha_{j m}
$$

The interpretation of $\alpha_{j m}$ by (24) is that the predictor's $x_{m}$ influence on the priority $\alpha_{j}$ equals the percent change of this priority $\partial \alpha_{j} / \alpha_{j}$ due to the percent change $\partial x_{m} / x_{m}$ in this predictor. It means that each parameter $\alpha_{j m}$ in (22) equals the elasticity of the priority $\alpha_{j}$ due to the varied predictor $x_{m}$. Therefore, depending on what is more convenient to use in a particular project - an absolute unit or a percent change in the predictors for evaluation of their impact on the priorities - the exponent (15) or CD (22) functions should be applied, respectively.

Although the ordinary least squares (OLS) regressions produce predictions independent of possible multicollinearity among the predictors, the analysis of the individual predictor importance and parameters of the model not prone to multicollinearity effects can be performed by special techniques, for example, described in (Lipovetsky, 2012; Lipovetsky \& Conklin, 2015b).

\section{Numerical comparisons for priority estimations}

Let us briefly describe the data from a marketing research project, and the modeling of the AHP priorities as functions of the auxiliary predictors performed in Lipovetsky (2021). Seventeen respondents compared flavors of a snack, and the AHP pairwise comparison ratios were elicited from each respondent for three newly introduced flavors (1 - A, 2 - E, and $3-\mathrm{H}$ ). The demographic variables for the respondents are: Gender, Age, number of people in the Household, Education, Employment, and Income, each represented by belonging to several designated groups. For example, the income groups are defined in thousand \$ as follows: 1 - less than 25; 2 - 25:39; 3 - 40:49; 4 - 50:74; 5 - 75:99; 6 - 100:124; 7 - 125:149; 8 - 150 or more.

The three pairwise ratios $a_{12}, a_{13}$, and $a_{23}$ of AHP matrices and six demographic variables are presented in 9 columns, by 17 observations (see Table 1 in Lipovetsky, 2021). Taking the elements $a_{12}, a_{13}$, and $a_{23}$ into one column yields the new dependent outcome of the pairwise ratios $y_{v}\left(v=1,2, \ldots\right.$, till all $3^{*} 17=51$ observations by responses). In the next columns, there are the corresponding binary indicators $\mathrm{A}$ and $\mathrm{H}$, where $\mathrm{A}$ shows by 1 that this item is in the numerator of the paired ratio, and by 0 that $\mathrm{A}$ is absent in the paired ratio; similarly, in $\mathrm{H}$ - zero means that it is not in the current pair comparison, and -1 if this item takes place in the denominator of the paired ratio. The item $\mathrm{E}$ is excluded as redundant because the total of the three columns $\mathrm{A}$, E, and $\mathrm{H}$ equals zero identically, so one of them is not needed. As mentioned after the formula (18), one set of the parameters should be put to zero for the uniqueness of the regression, and it corresponds to exclusion of the redundant indicator.

The demographic predictors are also taken in two sets, related to the items $\mathrm{A}$ and $\mathrm{H}$, as in the numerator and denominator in the ratios (16). The predictors related to the item A have positive or zero values exactly in correspondence with the values in the column A, showing that this item presented in the current paired ratio numerator or not. Similarly, the predictors related to the item $\mathrm{H}$ are taken with negative or zero values in correspondence with the values in the column $\mathrm{H}$, indicating that this item presented in the current paired ratio denominator or not. This design matrix is explicitly demonstrated in (Lipovetsky, 2021, Table 2). In that table, the set of demographic variables related to the item $A$ is denoted by the share number 1 (for example, gender.1, age.1, ..., income.1), and the set which goes with the item $\mathrm{H}$ is denoted by the share number 3. If instead of the exponential model the CD function is applied, the logarithms of the demographic values (22) constitute the variables in the design matrix described above.

In general, for $n$ compared items, there are $n(n-1) / 2$ pairwise ratios, and with $N$ respondents the total number of responses is $V=N n(n-1) / 2$. A combined model has $n-1$ sets of parameters, each includes the indicator (in our example, $\mathrm{A}$ or $\mathrm{H})$ plus $M$ auxiliary predictors, or $M+1$ parameters, so total $(n-1)(M+1)$ parameters. For building a regression, the total number of observations should be bigger than the total number of parameters, so such an 
Table 2. Cobb-Douglas regression model for AHP priority with demographic predictors.

\begin{tabular}{ccccc}
\hline & Estimate & Std. Error & $\mathrm{t}$ value & $\operatorname{Pr}(>|\mathrm{t}|)$ \\
\hline (Intercept) & -0.0077 & 0.2545 & -0.0301 & 0.9761 \\
A & -0.7938 & 1.0844 & -0.7321 & 0.4689 \\
H & -1.0094 & 1.0844 & -0.9308 & 0.3581 \\
gender.1 & 0.5447 & 0.4405 & 1.2366 & 0.2243 \\
age.1 & -0.0019 & 0.5361 & -0.0036 & 0.9972 \\
Household.1 & -0.3090 & 0.3080 & -1.0033 & 0.3224 \\
Education.1 & -0.0844 & 0.3844 & -0.2196 & 0.8274 \\
Employment.1 & 0.1885 & 0.2428 & 0.7765 & 0.4425 \\
Income.1 & 1.3361 & 0.2410 & 5.5437 & 0.0000 \\
gender.3 & -0.0576 & 0.4405 & -0.1307 & 0.8968 \\
age.3 & 0.2398 & 0.5361 & 0.4473 & 0.6573 \\
Household.3 & -0.0125 & 0.3080 & -0.0405 & 0.9679 \\
Education.3 & 0.3052 & 0.3844 & 0.7939 & 0.4324 \\
Employment.3 & 0.3348 & 0.2428 & 1.3792 & 0.1763 \\
Income.3 & 0.6978 & 0.2410 & 2.8954 & 0.0064 \\
R $^{2}$ & 0.7327 & & & \\
F-statistic & 7.05 & & & \\
p-value & $1.14 \mathrm{E}-06$ & & & \\
\hline
\end{tabular}

inequality should hold: $N n / 2>M+1$. In our example, $n=3, N=17$, and $M=6$, so this inequality is $25>7$, which is acceptable for regression modeling where at least three time more observations than the estimated parameters are commonly recommended.

The results of the linearized CD function (22) built by the described design matrix in one regression for both shares are presented in Table 2. In the first numerical column, there are estimates for the regression parameters of the priorities $\mathrm{A}$ and $\mathrm{H}$, and the demographics related to these first and third shares. The next columns contain the standard errors, t-statistics, and p-values of the coefficients. Only the income predictors are significant in the model for both shares. Below the parameters, there are characteristics of the model quality: the coefficient of multiple determination $R^{2}$, with the related $F$-statistics on 14 and 36 degrees of freedom, and its $p$-value. The value $R^{2}=0.7327$, and the square root of it as the coefficient of multiple correlation $R=0.8560$, suggest that the whole model is of a good quality of fit. $R^{2}$ has a meaning of the outcome variable's variance explained by the regression in comparison to its original variance relatively the mean level. By $R^{2}$ value of 73.27\% we can decide that this relative variability is good enough for all practical aims. What is more important - the Fisher F-test for the $R^{2}$ characteristic is big, and the corresponding $\mathrm{p}$-value is very small. All together it supports the conclusion about a good quality of the data fit with the CD model. It is interesting to note that the exponential model considered previously by the same data (Lipovetsky, 2021) has $R^{2}=0.6877$, thus, the CD function outperforms it. A quality of the model could be increased with more predictors, but there were no such variables in that marketing research study.

The parameters from Table 2 in two priorities (21) yield these CD functions:

$$
\begin{aligned}
& \alpha_{A}=e^{-0.7938}(\text { Gend })^{0.5447}(\text { Age })^{-0.0019}(\text { HH })^{-0.309}(\text { Educ })^{-0.0844}(\text { Empl })^{0.1885}(\text { Inc })^{1.3361} \\
& \alpha_{H}=e^{-1.0094}(\text { Gend })^{-0.0576}(\text { Age })^{0.2398}(\text { HH })^{-0.0125}(\text { Educ })^{0.3052}(\text { Empl })^{0.3348}(\text { Inc })^{0.6978}
\end{aligned}
$$

Using the CD functions (25) in their total (14), yields the shares of priorities of all three flavors for the predictors' values. Priorities dependance on a specific predictor can be studied by changing the values of this predictor and keeping the values of the other predictors on their mean level. Such profiling of priorities by the income is presented in Figure 2.

The regular AHP estimation of the constant priorities in this data produces the following values: $P_{A}=0.335$, $\mathrm{P}_{\mathrm{E}}=0.194$, and $\mathrm{P}_{\mathrm{H}}=0.471$. Therefore, the $\mathrm{H}$ and $\mathrm{E}$ are the most and least preferred flavors, respectively, so in general we can briefly write that $\mathrm{H}>\mathrm{A}>\mathrm{E}$. The graphs in Figure 2 reveal more interesting properties in the behavior of the flavors' preferences relatively their mean constant levels. With a growing income, the flavor A increases and the flavor E decreases noticeably, while the flavor $\mathrm{H}$ shows a concave behavior. Therefore, for a smaller income, the flavor $\mathrm{A}$ is the least preferable, and the order of priorities is $\mathrm{H}>\mathrm{E}>\mathrm{A}$. However, for the higher income, the 


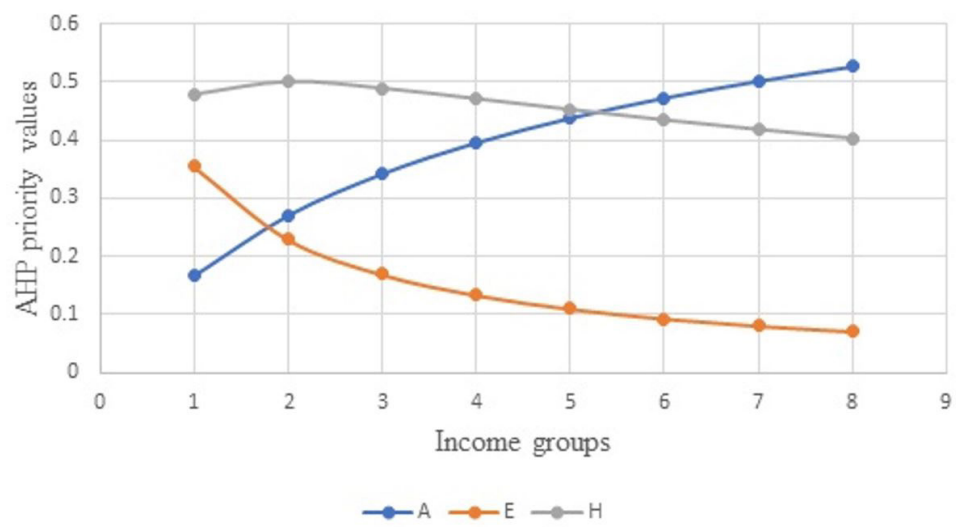

Figure 2. AHP priorities by Cobb-Douglas functions profiled by income.

flavor A becomes the most preferred, $\mathrm{A}>\mathrm{H}>\mathrm{E}$, overcoming even the item $\mathrm{H}$ at the income of approximately 80K. In general, behavior of these curves evaluated with CD functions, is similar to the previous finding by the exponential functions (Lipovetsky, 2021), but CD dependencies have more curvature in the change of priorities by the income predictor. Knowing these properties, the managers can take the adequate optimal decisions for advertising and promoting one or another product for different customers groups.

\section{Summary}

The paper considers the nonlinear scaling in the AHP approach to different problems, from the theoretical Two-Envelope Paradox to the applied priority modeling as functions by auxiliary predictors. The paper shows that that paradox can be easily dissolved if to use such scales, particularly, the logarithmic scale corresponding to the multiplicative utility function with the geometric mean for the expected utility value. Numerical comparison shows that both the shares and logarithmic scales are very close in a wide range of possible values of the ratios.

These scales are useful in other applications of the multiple-criteria decision making as well, particularly, in the AHP vectors of priority evaluation. For multiple respondents with different demographic and socio-economic properties, the priority vectors can be estimated not as constants but as regression models by these auxiliary predictors. Prediction of the priorities by these models can be made for individual respondents, and profiled by the predictors. Numerical results are encouraging - they show that the considered methods present useful features of priorities behavior.

Summarizing the main results, it is possible to state that the nonlinear scaling is valuable theoretically, for example, giving a solution to the Two-Envelope problem, and is useful in practical AHP priority estimation, where it helps to decision makers to focus on the clients' individual needs, to elaborate on specific promotion of products to customers in accordance with their demographics, socio-economics and various other characteristics measured by the auxiliary predictors.

The future studies can be seen in different directions, for example, with a panel data elicited in different moments in time, when the preferences of the alternatives can vary and be forecasted in time. There are other problems, for instance, of the choice depending on the fluctuating prices, where the preferences among items depend on changing conditions. A challenging problem can also be seen in the synthesizing the priority vectors obtained in the functional forms by different levels of the hierarchy from the items' comparisons within each criterion to the criteria comparisons among themselves into the global priorities expressed as functions by all the auxiliary variables.

\section{Acknowledgements}

1 am grateful to two reviewers whose comments and suggestions helped to improve the paper.

\section{References}

Abbott, D., Davis, B. R., \& Parrondo, J. M. (2010). The two-envelope problem revisited. Fluctuation and Noise Letters, 9(1), 1-8. http:// dx.doi.org/10.1142/S0219477510000022. 
Albers, C. J., Kooi, B. P., \& Schaafsma, W. (2005). Trying to resolve the two-envelope problem. Synthese, 145(1), 89-109. http://dx.doi. $\operatorname{org} / 10.1007 / \mathrm{s} 11229-004-7665-5$.

Egozcue, M., \& Fuentes García, L. (2015). An optimal threshold strategy in the two-envelope problem with partial information. Journal of Applied Probability, 52(1), 298-304. http://dx.doi.org/10.1239/jap/1429282624.

Ishizaka, A., Tasiou, M., \& Martínez, L. (2020). Analytic hierarchy process-fuzzy sorting: An analytic hierarchy process-based method for fuzzy classification in sorting problems. The Journal of the Operational Research Society, 71(6), 928-947. http://dx.doi.org/10 $.1080 / 01605682.2019 .1595188$.

Keeney, R. L., \& Raiffa, H. (1993). Decisions with multiple objectives: preferences and value tradeoffs. New York: Cambridge University Press. http://dx.doi.org/10.1017/CB09781139174084.

Lee, C. (2013). The Two-envelope Paradox: asymmetric cases. Mind, 122(485), 1-26. http://dx.doi.org/10.1093/mind/fzt023.

Lipovetsky, S. (2009). Global priority estimation in multiperson decision making. Journal of Optimization Theory and Applications, 140(1), 77-91. http://dx.doi.org/10.1007/s10957-008-9447-6.

Lipovetsky, S. (2012). Interpretation of Shapley Value regression coefficients as approximation for coefficients derived by elasticity criterion. In Proceedings of the Joint Statistical Meeting of the American Statistical Association (JSM'12) (pp. 3302-3307). San Diego, CA.

Lipovetsky, S. (2019). Express analysis for prioritization: best-worst scaling alteration to System 1. Journal of Management Analytics, 71), 12-27. http://dx.doi.org/10.1080/23270012.2019.1702112.

Lipovetsky, S. (2020a). AHP and Two-Envelope Paradox resolution via nonlinear scaling. In Proceedings of the International Symposium on Analytic Hierarchy Process. Pittsburgh, PA, USA. http://dx.doi.org/10.13033/isahp.y2020.007.

Lipovetsky, S. (2020b). Personalized key drivers for individual responses in regression modeling. International Journal of Risk and Contingency Management, 9(3), 15-30. http://dx.doi.org/10.4018/1JRCM.2020070102.

Lipovetsky, S. (2020c). Priority vector estimation: consistency, compatibility, precision. International Journal of the Analytic Hierarchy Process, 12(3), 577-591. http://dx.doi.org/10.13033/ijahp.v12i3.801.

Lipovetsky, S. (2021). Predictor analysis of group decision making. Stats, 4(1), 108-121. http://dx.doi.org/10.3390/stats4010009.

Lipovetsky, S., \& Conklin, M. (2015a). AHP priorities and Markov-Chapman-Kolmogorov Steady-States probabilities. International Journal of the Analytic Hierarchy Process, 72), 349-363. http://dx.doi.org/10.13033/ijahp.v7i2.243.

Lipovetsky, S., \& Conklin, M. (2015b). Predictor relative importance and matching regression parameters. Journal of Applied Statistics, 42(5), 1017-1031. http://dx.doi.org/10.1080/02664763.2014.994480.

Lipovetsky, S., \& Tishler, A. (1999). Interval estimation of priorities in the AHP. European Journal of Operational Research, 114(1), 153164. http://dx.doi.org/10.1016/S0377-2217(98)00012-5.

Lootsma, F. (1999). Multi-criteria decision analysis via ratio and difference judgement. London: Kluwer Academic Publishers. http:// dx.doi.org/10.1007/b102374.

Markosian, N. (2011). A simple solution to the Two Envelope problem. Logos \& Episteme, 2(3), 347-357.

McDonnell, M. D., Grant, A. J., Land, 1., Vellambi, B. N., Abbott, D., \& Lever, K. (2011). Gain from the two-envelope problem via information asymmetry: on the suboptimality of randomized switching. Proceedings of the Royal Society of London. Series A, 4672134), 2825-2851. http://dx.doi.org/10.1098/rspa.2010.0541.

Neira-Rodado, D., Ortíz-Barrios, M., De la Hoz-Escorcia, S., Paggetti, C., Noffrini, L., \& Fratea, N. (2020). Smart product design process through the implementation of a fuzzy Kano-AHP-DEMATEL-QFD approach. Applied Sciences, 10(5), 1792. http://dx.doi. org/10.3390/app10051792.

Noto, S., Shiroiwa, T., Kobayashi, M., Murata, T., lkeda, S., \& Fukuda, T. (2020). Development of a multiplicative, multi-attribute utility function and eight single-attribute utility functions for the Health Utilities Index Mark 3 in Japan. Journal of Patient-Reported Outcome, 4(1), 23. http://dx.doi.org/10.1186/s41687-020-00188-8. PMid:32246218.

Ramos, M. O., Silva, E. M., \& Lima-Júnior, F. R. (2020). A fuzzy AHP approach to select suppliers in the Brazilian food supply chain. Production, 30, 1-16. http://dx.doi.org/10.1590/0103-6513.20200013.

Saaty, T. L. (1980). The analytic hierarchy process. New York: McGraw-Hill.

Saaty, T. L., \& Peniwati, K. (2007). Group decision-making: drawing out and reconciling differences. Pittsburgh, PA: RWS Publications.

Saaty, T. L., \& Vargas, L. G. (1984). Comparison of eigenvalue, logarithmic least squares and least squares methods in estimating ratios. Mathematical Modelling, 5(5), 309-324. http://dx.doi.org/10.1016/0270-0255(84)90008-3.

Whitaker, R. (2007). Validation examples of the Analytic Hierarchy Process and Analytic Network Process. Mathematical and Computer Modelling, 46(7-8), 840-859. http://dx.doi.org/10.1016/j.mcm.2007.03.018.

Yang, J., Vargas, L. G., Jin, X., \& Zhou, L. (2020). Quality credit evaluation in the internet company: a system based on the analytic hierarchy process. Journal of Business Economics and Management, 21(2), 344-372. http://dx.doi.org/10.3846/jbem.2020.11409.

Yi, B. U. (2013). Conditionals and a Two-envelope Paradox. The Journal of Philosophy, 110(5), 233-257. http://dx.doi.org/10.5840/ jphil2013110527. 